\title{
Simple or Simplistic?
}

\author{
Patrick Clifford
}

Consideration of the symposium's title, On Adams House in the Pacific, brought to mind questions around the rhetoric of "simple or simplistic?" We have often felt that we walked a tightrope between those poles, as we sought to make rigorous and clear architecture, beautiful but not pretty, and challenging but not challenged. We have described our work, to ourselves at least, as dense (i.e., simple, but not simplistic).

We come from the "Man alone" tradition in New Zealand and some of our early projects, in particular, were small and often idealised versions of 'the bach'. This work continues in more recent, similar projects, and its influence even extends to the design of what we might call sheds, rather than huts. The striving for a nonsimplistic simplicity has informed not only our approach to the 'product', but also to the process of building. When thinking about the sequence of work resulting from our designs, we have endeavoured to create sequences which would benefit and enable the site, and make the process smooth and efficient: in the tradition of lightweight building culture, the roof is constructed on the ground (whole or in parts) and then erected to provide shelter for the processes to follow.

\section{House at Great barrier I (1993)}
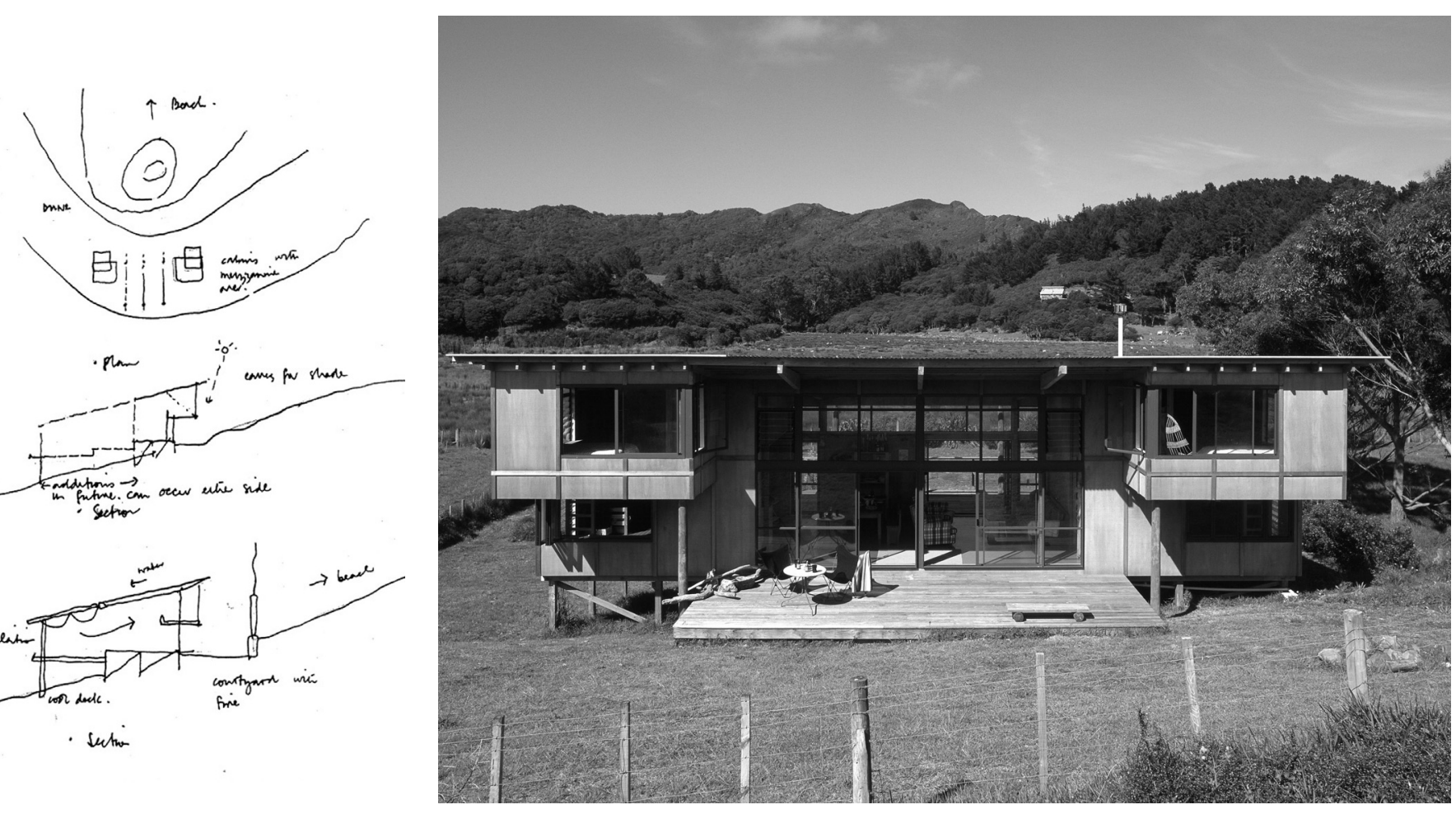


\section{House at Te Horo (1994)}


A variation on a theme - two small cabins, this time living, organised on a wooden platform defining an outdoor living space. Photo: Simon Devitt

\section{House at Great Barrier II (2008)}
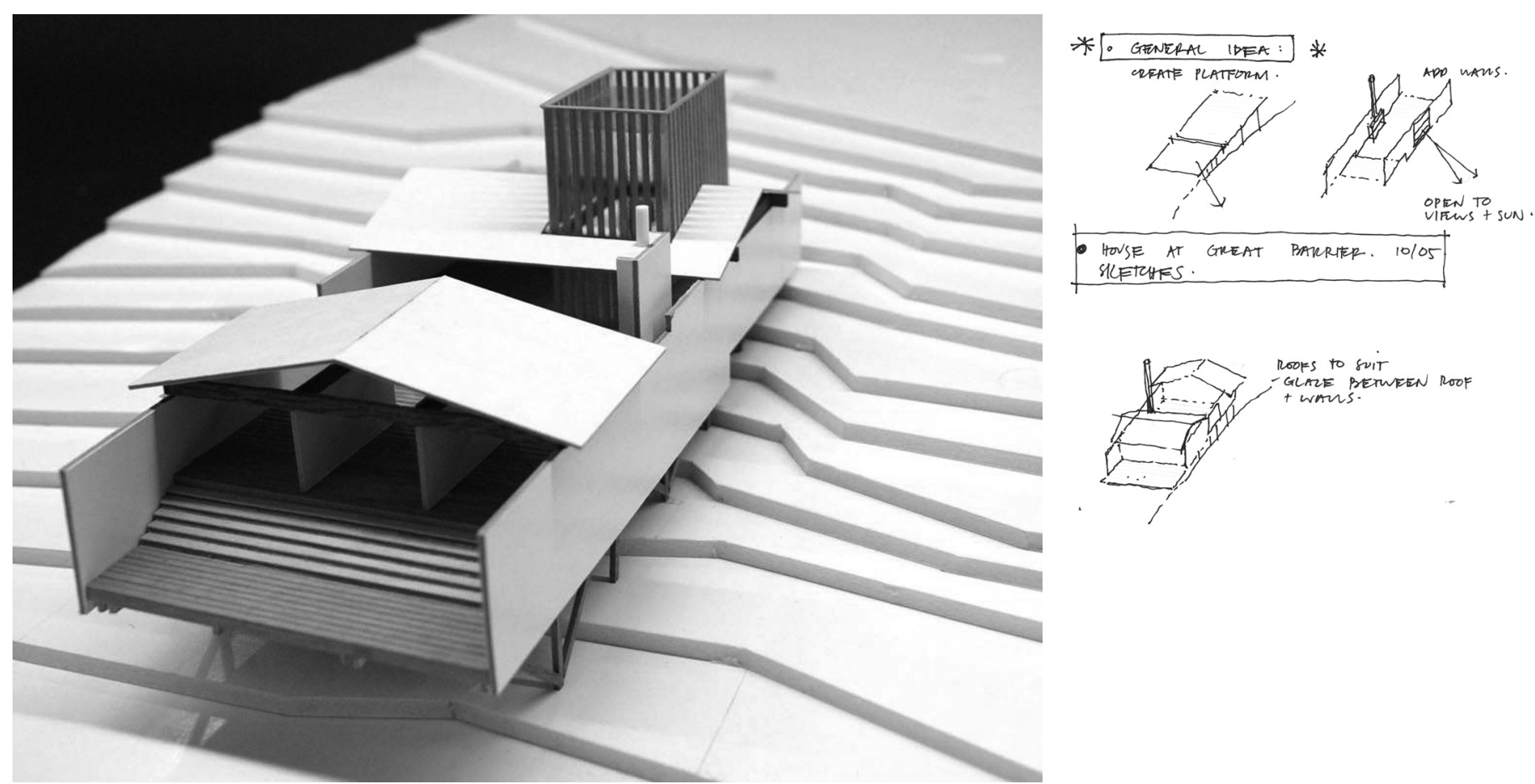

Two small cabins - for two families or groups - organised on and around a wooden platform. The cabins define a shared living space, the whole covered by a skillion roof. Photo: Patrick Reynolds 
Wairarapa House (2007)

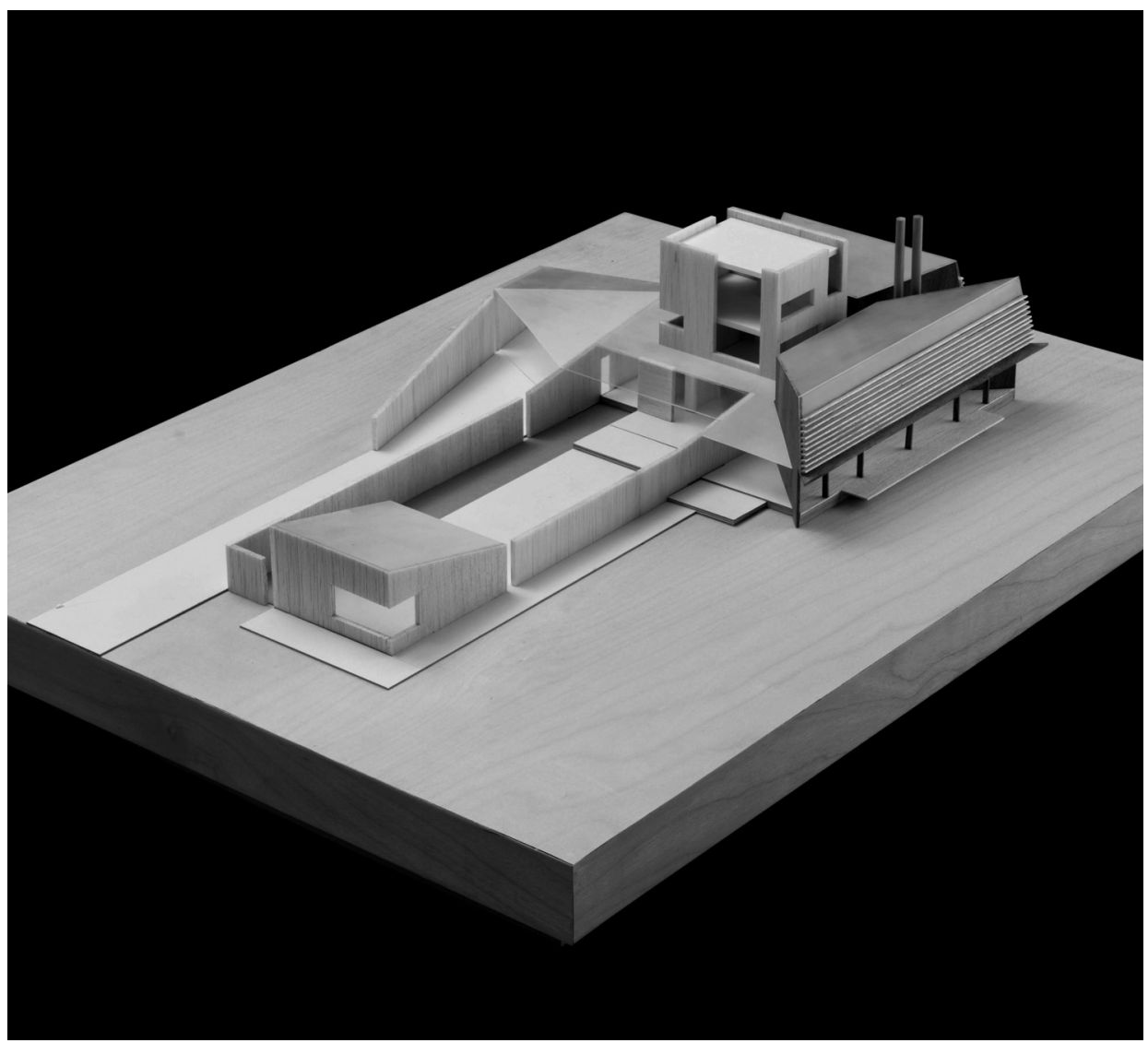

A further variation - two sleeping areas, lightweight in construction, define a living space under a skillion roof. The clients' suggestion of a walled garden is translated into a connecting element that further organises the project, adding weight and definition both in the front and in the back. Photo: architectus auckland

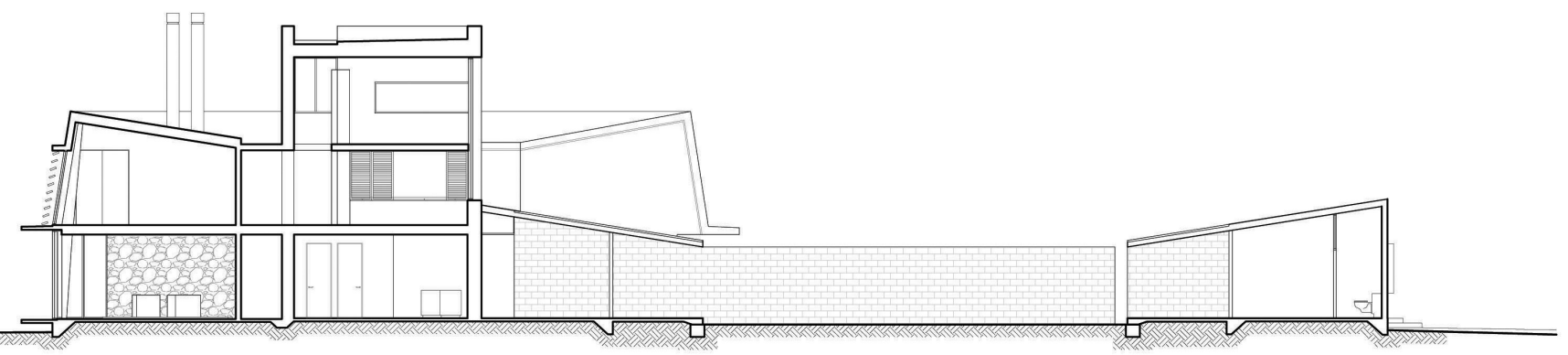

The design explores the relationship between heavy walls - in this case enclosing a courtyard, a farmyard and a small tower - and lightweight rooms. The programme is more extensive than in the Te Horo House and the material roles reversed: in general terms, the living spaces are light, whilst the bedrooms are massive. Photo: architectus auckland 


\section{Engineering and Science Research Centre ( 200I)}

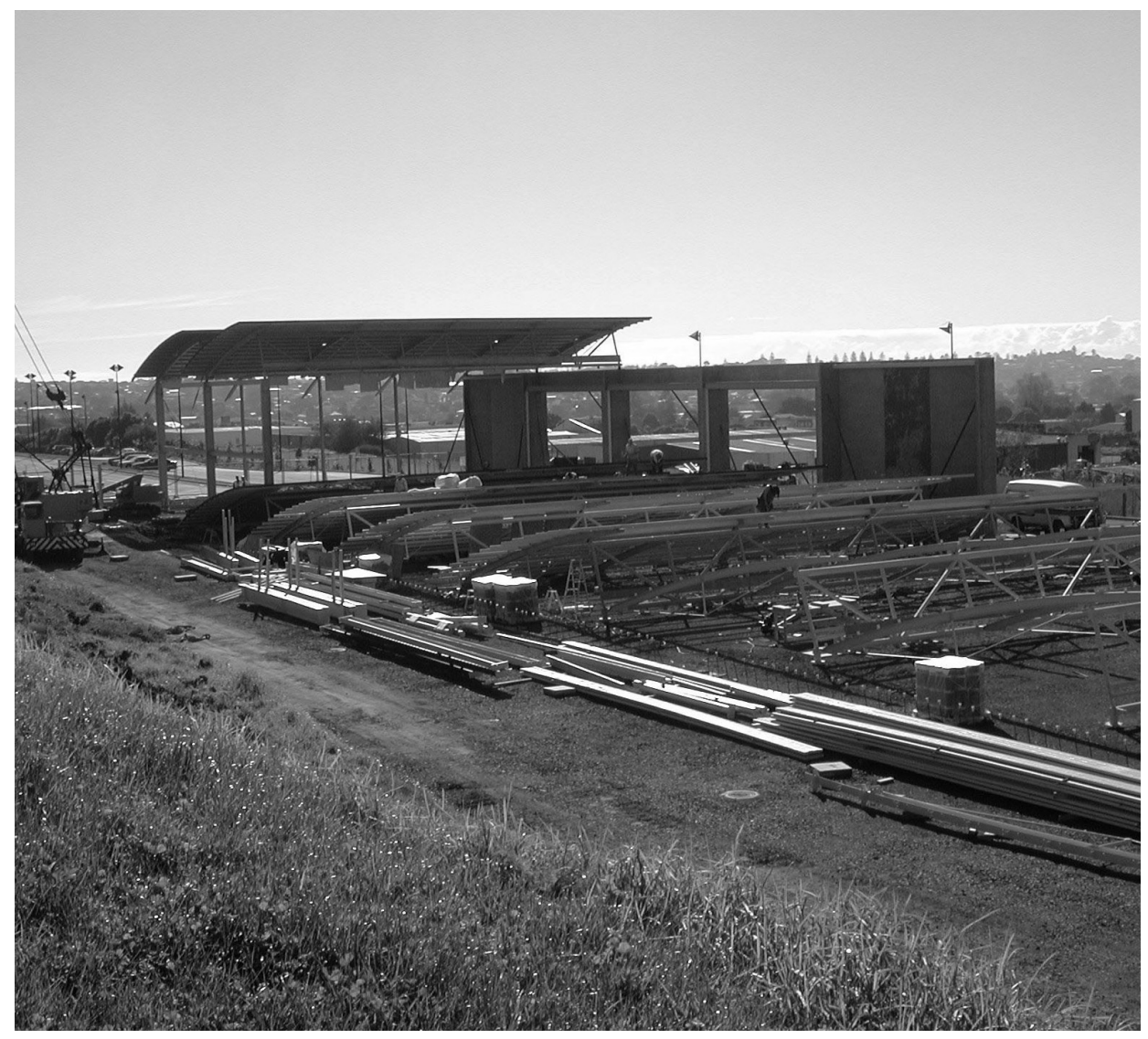

A South light shed $-100 \mathrm{~m}$ long and $20 \mathrm{~m}$ wide, with an attached $10 \mathrm{~m}$ wide bar of service spaces and a colonnade. The $10 \mathrm{~m}$-wide roof elements are assembled on the ground, complete with the waterproof membrane, and then craned into place .The concrete slab is poured and the walls constructed after this shelter is made. Photo: architectus auckland
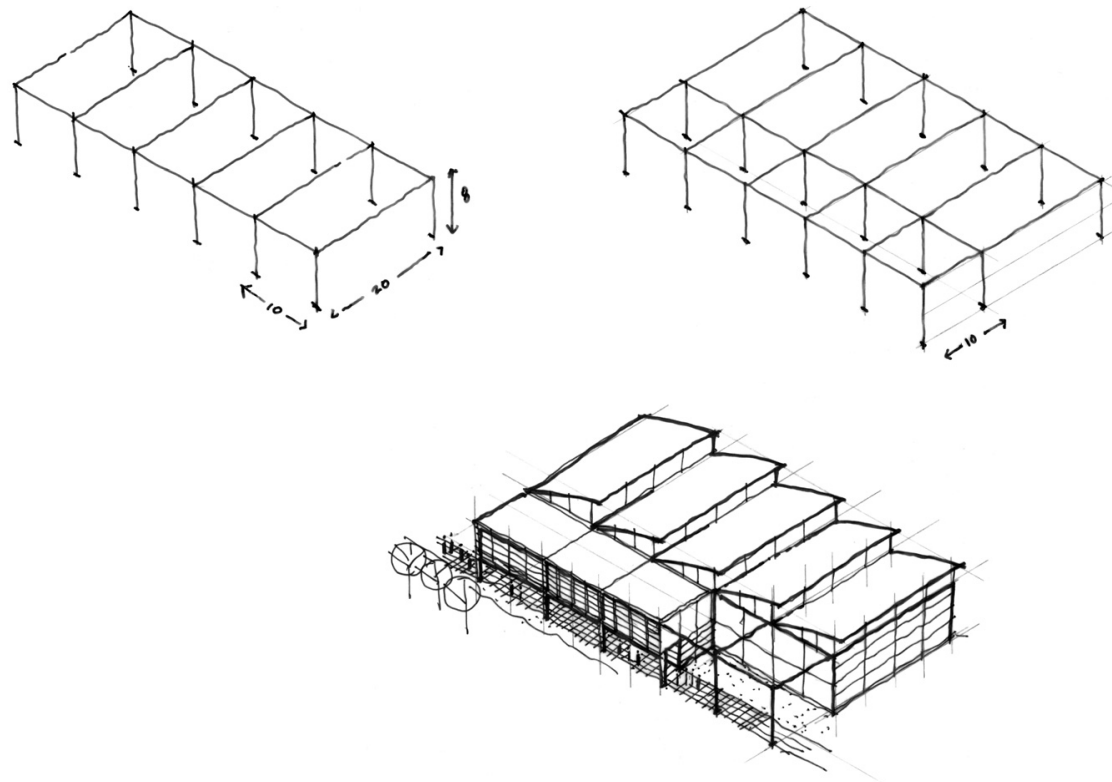


\section{Auckland Grammar Sports Centre (2007)}



Another variation on a theme - working with the same contractor, a $36 \mathrm{~m} \times 36 \mathrm{~m}$ roof element was again constructed on the ground, then finished and craned into place. Everything getting bigger and heavier means more cranes and less tolerance. The slab is poured in advance, and the corners are filled in when the erection is complete. Photo: architectus auckland



